\title{
Dynamic Adverse Selection with a Patient Seller
}

\author{
Juan Beccuti, Marc Möller*
}

Department of Economics, University of Bern. Schanzeneckstrasse 1, 3001 Bern, Switzerland.

\begin{abstract}
This paper considers dynamic bilateral trade with short-term commitment. We show that, when the seller is more patient than the buyer, there exist systematic differences between the optimal selling and renting mechanisms. While the former consists of simple price-posting, the latter induces the buyer to choose between a secure- and a random-delivery contract. Allowing for mechanisms more general than price-posting reduces the seller's cost of learning the buyer's valuation in the renting case. Renting leads to more learning than selling but (unless the horizon is sufficiently long) only when general mechanisms are available. Our results contrast with the common view that the restriction to price-posting is innocuous and that informational asymmetries are more persistent under renting than under selling.
\end{abstract}

Keywords: Dynamic adverse selection, mechanism design, price-posting.

JEL: D82, D86, D42.

${ }^{*}$ Corresponding author.

E-mail addresses: juan.beccuti@vwi.unibe.ch, marc.moeller@vwi.unibe.ch 


\section{Introduction}

In one of the most fundamental economic transactions, a monopolistic seller offers an indivisible durable product to a single (non-anonymous) buyer whose valuation constitutes his private information. An important insight of the literature on dynamic adverse selection holds that the seller's inability to commit to the future terms of trade represents an obstacle for learning. Fudenberg and Tirole (1983) and Sobel and Takahashi (1983) show that, without commitment, the seller will offer a decreasing sequence of prices, inducing him to learn the buyer's type only gradually. When the product is rented rather than sold, informational asymmetries turn out to be even more persistent. Hart and Tirole (1988) argue that, due to the so-called ratchet effect, the seller cannot do better than by offering a rental price so low as to prevent himself from learning anything about the buyer's type for most of the time horizon.1

More recent results suggest that allowing for more sophisticated mechanisms than simple price posting does not alter these conclusions. Indeed, using a selling model with a continuum of types, Skreta (2006) finds that price-posting constitutes the seller's optimal selling mechanism. In this paper, we argue that in the renting case, learning can be improved by use of a mechanism whose outcome is not implementable via simple priceposting. In particular, we show that if general mechanisms are available, the seller's optimal renting mechanism is random and induces more learning than the seller's optimal selling mechanism. These results come at a surprise as they contrast with the view, suggested by the existing literature, that the restriction to price-posting is innocuous and that informational asymmetries are more persistent under renting than under selling.

Our setting resembles Hart and Tirole's (1988) seminal model of dynamic bilateral trade with short-term commitment. In each of a finite number of periods, the buyer has a

\footnotetext{
${ }^{1} \mathrm{~A}$ similar conclusion is obtained by the dynamic incentive models of Laffont and Tirole $(1987,1988)$ which bear a certain resemblance to the renting framework of Hart and Tirole (1988). They show that full separation is not feasible (for a continuum of types) or might be dominated by pooling (for a binary type).
} 
unit demand for the seller's product. The buyer's per-period valuation can be either high or low and remains constant across periods. Our analysis encompasses both the renting and the selling case. While in the former, the seller offers his product to the buyer in every period, in the latter the interaction ends once a delivery has taken place. We differ from Hart and Tirole (1988) in that we choose a general mechanism design approach.2 At the start of each period, the seller commits to a mechanism which, due to his lack of long-term commitment, must be optimal given his (potentially updated) belief about the buyer's type. A mechanism specifies a probability with which the product is delivered to the buyer and a transfer from the buyer to the seller, both conditional on the buyer's message.

Our analysis focuses on the case where the seller is more patient than the buyer. From a theoretical perspective, this case turns out to be the interesting one to consider. It is relevant from an applied viewpoint because in many markets sellers (firms) adopt a more long-term perspective or have access to cheaper credit than buyers (consumers) 3

Applying the revelation principle of Bester and Strausz (2001), we find that with a patient seller there exists a range of (moderate) prior beliefs for which the seller induces the buyer to separate (in the first period) although pooling would be optimal in a static context. The reason for this result is straightforward and the same as in the price-posting models of Fudenberg and Tirole (1983) and Sobel and Takahashi (1983). When the seller is more patient, information about the buyer's type is more valuable to the seller than to the buyer, giving the seller an incentive to separate types even at the cost of a sub-optimal

\footnotetext{
${ }^{2}$ Their model allows for general mechanisms in the analysis of long-term contracting with renegotiation, but in their treatment of the non-commitment case they "restrict attention to deterministic offers" (Hart and Tirole (1988), p.512).

${ }^{3}$ Although a common assumption in bargaining models (e.g., Fudenberg and Tirole (1983), Sobel and Takahashi (1983)), the literature on dynamic mechanism design has abstracted from heterogeneous discounting. Heterogeneous discounting makes dynamic contracting problems ill-defined when parties are able to commit to inter-temporal transfers (Krähmer and Strausz, 2015). In order to facilitate comparisons with the commitment case (as in Hart and Tirole, 1988), the literature has maintained the assumption of homogeneous discounting even when, as in this paper, parties are unable to commit to future payments.
} 
allocation.

Perhaps surprisingly, heterogeneous discounting generates systematic differences between the optimal renting and selling mechanisms that are absent when discount factors are the same. In particular, we find that the optimal renting mechanism consists of a menu containing a secure-delivery and a random-delivery contract whereas the optimal selling mechanism can be implemented through simple price-posting. Allowing the seller to offer random contracts can lead to a significant increase of more than $10 \%$ in the seller's payoff from renting relative to what he can achieve by simple price-posting (see Figure 1) 4

To understand why random contracts are optimal in the renting setting, an important insight is that the presence of the ratchet effect requires the low type's (probability of) trade to fall short of the high type's trade by a strictly positive amount, for separation to be feasible. 5 Hence, when the seller chooses to separate, he will set the low type's likelihood of delivery equal to its upper bound, resulting in an outcome that is unattainable through simple price-posting. By doing so, the seller minimizes his loss from implementing a suboptimal trade with the low type. Random-delivery contracts are useful in the renting case because they allow the seller to elicit the buyer's type without excluding low valuation buyers from trade altogether. This can be understood most easily in the limiting case of a myopic buyer. In this case, the ratchet effect vanishes and separation is achievable through a menu with the low type's probability of delivery chosen smaller but arbitrarily close to one. With a myopic buyer, random contracts allow the seller to learn the buyer's type at negligible cost.

When we compare renting and selling with respect to payoffs, a straightforward but

\footnotetext{
${ }^{4}$ In reality, the seller may induce randomness into his contracts by conditioning on exogenous stochastic events. For example, maintenance contracts for IT or other industrial equipment often restrict their service to weekdays (as opposed to 24/7). If a breakdown occurs on a weekend the buyer of the service derives no value from the contract.

${ }^{5}$ It will become clear that this requirement, reminiscent of the monotonicity constraint in the static context, is necessary to induce both types of buyers to reveal their type truthfully (with positive probability).
} 
important observation is that for a patient seller, renting is preferable over selling. The simple reason is that selling requires the seller to charge the buyer for the future use of his product upfront. The dominance of renting over selling for a patient seller implies that we have identified price-posting as sub-optimal in a situation where it actually matters. In particular, when a seller is free to choose between renting and selling and general mechanisms are available, then our model predicts that mechanisms more sophisticated than simple price-posting will be observed (for some parameter values).

Our analysis concludes with a comparison between renting and selling with respect to the persistence of informational asymmetries. We find that the range of priors for which the seller pools both types of buyer for the entire trade-horizon is smaller under renting than under selling. Notably, (for short horizons) this conclusion hinges on the availability of random contracts. When the seller is restricted to simple price-posting, the result is reversed, i.e., renting leads to more pooling than selling. This result is driven by the fact that, under renting, learning is not only more beneficial but also more costly than under selling. Allowing for general mechanisms lowers the costs of learning in the renting case, making learning become more desirable than under selling. This finding highlights the importance of allowing for general mechanisms in models of dynamic bilateral trade with short-term commitment.

The plan of the paper is as follows. Following a discussion of the related literature and the description of the model (Section 2) we provide a motivating example (Section 3) that serves to highlight our subsequent results. Section 4 posits the seller's general mechanism design problems for the renting and the selling case. We derive the corresponding reduced programs, containing the dynamic equivalent of the monotonicity constraint, familiar from the static case. In Section 5 we characterize and compare the seller's optimal renting and selling mechanisms when there are only two periods of (potential) trade. The two-period case serves as the starting point for our inductive analysis of the general case contained in Section 6. Section 6, besides proving robustness, serves to highlight the optimality of 
a novel kind of semi-separating mechanism that emerges in the renting case when there are more than two periods of trade. This mechanism prevents the seller's belief about the buyer's type from becoming too optimistic too early, allowing the seller to distribute his learning across periods. While our analysis focuses on the (more interesting) case of a soft seller, characterized by a low prior belief about the buyer's type, we have confirmed that our main insight that renting reveals more information than selling survives when the seller is tough.

\section{Related literature}

This paper contributes to the literature on dynamic adverse selection, initiated by the regulation model of Freixas et al. (1985). A common theme in this literature is that, in the absence of long-term commitment, the ratchet effect obstructs information from being revealed (e.g., Hart and Tirole, 1988). While it may not be surprising that the buyer's impatience mitigates the ratchet effect, the key observation of our paper is that heterogeneous discounting induces renting contracts to be random whereas in a selling framework price-posting remains optimal.

The random allocation of an indivisible product can be interpreted as the supply of an interior quantity of a divisible good. This connects our paper to the models of dynamic adverse selection with continuous allocations, pioneered by Laffont and Tirole (1990). This literature challenges the view that separation requires commitment. If the seller can offer different quantities of his product, time ceases to be the only screening variable available. Wang (1998) recognizes that with the help of an additional screening variable, a seller may not suffer from his inability to commit to future offers as conjectured by Coase (1972). By offering a menu of contracts specifying differing quantities, the seller can separate the buyer without delay. In the unique sequential equilibrium of Wang's infinite horizon model, full and immediate separation results from the offer of the optimal static 
screening menu in the first period 6 In our model, heterogeneous discounting changes the relative slope of the players' iso-utility curves across periods, encouraging the parties to "trade information inter-temporally" 7 As a consequence, separation via "quantity" emerges in a dynamic context, even when the optimal static contract would pool the buyer by offering the same (non-random) allocation to both types. Under renting, quantity is a more effective screening variable than under selling, because the inefficiency resulting from the downward distortion of the low type's quantity is restricted to the current period.

Whether quantity can substitute for time as a screening device is less clear when contracts are subject to renegotiation. In particular, the seller and the buyer may find it mutually beneficial to increase quantity towards its efficient level once the seller has learned the buyer's type. Maestri (2017) and Strulovici (2017) show that the possibility of renegotiation erodes the seller's ability to screen the buyer and restores efficient allocations in the limit as negotiation-frictions vanish. While in our selling model "renegotiation" only happens when the product failed to be allocated to the buyer, in our renting model a new "quantity" is negotiated in every period due to the seller's lack of long-term commitment. In this sense, there exists more opportunity for renegotiation under renting than under selling and our result that (for longer horizons) semi-separation arises as a feature of the optimal renting mechanism resonates well with the literature on dynamic contracting with renegotiation.

Random delivery contracts have been shown to be useful as a screening device in several related but distinct frameworks. Thanassoulis (2004) shows that it may be optimal for the seller of two substitute goods to offer his customers a product lottery inducing the delivery of a random product. Buyers with large differences in their valuations are willing to pay a higher price for obtaining their desired product with certainty. Manelli and

\footnotetext{
${ }^{6}$ Gerardi and Maestri (2016) find immediate and full information revelation in an infinite horizon model where in each period the informed party has the choice between staying in the relationship or leaving forever to claim an outside option.

${ }^{7}$ We are grateful to the anonymous associate editor for pointing out this analogy.
} 
Vincent (2007) and Pavlov (2011) extend this insight to the case of an arbitrary number of (not necessarily substitute) products where, besides randomness, bundling can play a role. A new feature that arises when products are not substitutes is the introduction of uncertainty not only about the identity of the delivered product but also about whether or not a delivery takes place at all. This is the kind of randomness we find to be optimal in our setting. However, as explained nicely by Hart and Reny (2015), randomness arises in these settings precisely because of the existence of multiple products. In our setting with a single product, random mechanisms turn out to be optimal because trade is dynamic rather than static.

Finally, our model allows for the interpretation of random-delivery contracts as "damaged products" in the spirit of Deneckere and McAfee (1996). This connects our model to the literature on monopolistic screening with a quality dimension initiated by Mussa and Rosen (1978). The special feature of our model is that the "costs" of supplying different levels of quality are the same (zero) for all qualities below a certain maximum. It is important to note that with this cost structure, intermediate levels of quality cannot be part of the seller's optimal static menu. In our setting, the randomness of the optimal contract is an intimate consequence of the dynamic nature of contracting.

\section{Model}

We consider a buyer and a seller who interact for $N \geq 2$ periods. The seller offers an indivisible durable product and the buyer has a unitary demand. For simplicity, we abstract from production costs 8 Our model distinguishes between two cases. In the renting case, the product is allocated to the buyer for only one period at a time. In the selling case, the product is allocated to the buyer for all remaining periods.

The buyer's (per-period) valuation of the good, $\theta$, is constant across periods. It can

\footnotetext{
${ }^{8}$ All results remain valid when the seller has a constant unit cost of production sufficiently small for trade to be efficient independently of the buyer's valuation.
} 
take a high value normalized to $\theta_{H}=1$ or a low value $\theta_{L} \in(0,1)$. For brevity we define $\Delta \theta \equiv 1-\theta_{L}$. The buyer's valuation $\theta$ constitutes his private information and will therefore be denoted as his type. We let $\beta \in(0,1)$ be the seller's (prior) belief about the buyer's probability of having a high type. Our analysis focuses on the case where, in the language of Fudenberg and Tirole (1983), the seller is soft. A soft seller, being characterized by a sufficiently low prior, $\beta<\theta_{L}$, would sell to both types of buyer at a low price if there was only one period of trade 9

If in any given period, the buyer makes a transfer $t$ to the seller then the buyer's (instantaneous) payoff is given by $\theta-t$ if the product is allocated to him. If the product is not allocated to the buyer during that period, his payoff is given by $-t$. The seller's (instantaneous) payoff is equal to his revenue $t$. Buyer and seller discount future payoffs with discount factors $\delta_{B} \in(0,1)$ and $\delta_{S} \in(0,1)$, respectively. We focus on the case where the seller is more patient than the buyer by assuming that $\delta_{S}>\delta_{B}$. It will become clear below that, in our setting, $\delta_{S}>\delta_{B}$ represents the "interesting case", whereas for $\delta_{S} \leq \delta_{B}$ the model's predictions remain the same as under homogeneous discounting. Moreover, we will see that, in the renting case, (full) separation is not feasible when the buyer values future payoffs too strongly (see explanation following Lemma 1). In order to study the optimality of separation in a setting where separation is feasible we make the following:

\section{Assumption 1.}

$$
\sum_{n=1}^{N-1} \delta_{B}^{n}<1 .
$$

Note that Assumption 1 holds for all $N$ when $\delta_{B}<\frac{1}{2}$ and is automatically satisfied for $N=2$.

While earlier articles on dynamic adverse selection have analyzed settings with longterm commitment, the focus of the literature has shifted to the (more realistic but also

\footnotetext{
${ }^{9}$ For a tough seller, $\beta \geq \theta_{L}$, the optimal mechanisms are qualitatively identical to the homogeneous discounting case. In particular, as we will argue in Section 5, price-posting is optimal both under renting and selling. See also footnote 10 .
} 
more complicated) case in which no such commitment is available. We follow this tendency by assuming that, in any given period, the seller can commit to the "terms of trade" for that period but cannot commit to the future "terms of trade". Using Perfect Bayesian Equilibium as our solution concept, this assumption implies that the seller's offer in any period must be (sequentially) optimal given the trading history up to that period.

Besides our assumption of short-term commitment, we put no restrictions on the way in which the seller and the buyer interact. In particular, we do not restrict attention to the possibility that the seller may simply post a price for his product. Instead, we use a mechanism design approach to determine the seller's revenue maximizing strategy. Following Bester and Strausz (2001), we can assume without loss of generality, that in any given period the seller offers a direct mechanism in which the buyer is asked to report his type and, conditional on his message $m \in\{l, h\}$, the mechanism specifies an outcome consisting of a transfer $t_{m} \in \Re$ from the buyer to the seller and a likelihood $x_{m} \in[0,1]$ with which the product is allocated to the buyer. We say that a mechanism's outcome can be implemented by price-posting when $x_{l}, x_{h} \in\{0,1\}$ and there exists a price $p$ such that $t_{m}=p x_{m}$ for any $m \in\{l, h\}$.

\section{Motivating example}

In this section we motivate our subsequent analysis by way of an example. The example focuses on the renting case in order to demonstrate the usefulness of random-delivery contracts. It assumes that there are $N=2$ periods of trade and that the seller believes the buyer's valuation for his product to be $\theta_{H}=1$ with probability $\beta=30 \%$ and $\theta_{L}=1 / 2$ with probability $1-\beta=70 \%$. The seller's discount factor is assumed to be given by $\delta_{S}=\frac{3}{4}$, whereas the buyer's discount factor is $\delta_{B}=\frac{1}{4}$.

Suppose first that the seller's strategy consists of posting a price in each period. If he posts a low price $p=1 / 2$ in period 1 , it will be accepted by both types. In this case, the 
seller learns nothing from his observation of the buyer's first period behavior and given his prior he optimally chooses $p=1 / 2$ again in period 2. Following this strategy, the seller's payoff is $1 / 2+\frac{3}{4} \cdot 1 / 2=7 / 8$.

Alternatively, the seller may post a high price in period 1 that is accepted only by the high type. As this allows the seller to learn the buyer's type, the seller will set the period 2 price equal to the buyer's true valuation. In order to make the high type accept in period 1 the seller has to compensate him for facing a high price $p=1$ rather than a low price $p=1 / 2$ in period 2 by charging $p=1-\frac{1}{4}(1-1 / 2)=7 / 8$ in period 1 . Following this strategy, the seller's payoff is $30 \% \cdot\left(7 / 8+\frac{3}{4} \cdot 1\right)+70 \% \cdot \frac{3}{4} \cdot 1 / 2=6 / 8<7 / 8$. Hence when the seller's strategy is restricted to simple price-posting, he will refrain from inducing the buyer to reveal his type.

What if we allow the seller to use more sophisticated renting mechanisms? Consider the possibility that in period 1 the seller offers the buyer the following choice: Make a transfer of $t_{h}=1 / 2$ and obtain the product with probability $x_{h}=1$ or make a smaller transfer of $t_{l}=3 / 8$ and obtain the product only with probability $x_{l}=0.75$. This menu makes it optimal for the high and the low type to choose secure- and random-delivery respectively. To see that the high type buyer has no incentive to pool with the low type note that his payoff from random-delivery $0.75 \cdot 1-3 / 8+\frac{1}{4}(1-1 / 2)$ is just the same as his payoff from secure-delivery $1-1 / 2=1 / 2$. The seller's payoff is $30 \% \cdot\left(1 / 2+\frac{3}{4} \cdot 1\right)+$

$70 \% \cdot\left(3 / 8+\frac{3}{4} \cdot 1 / 2\right)=72 / 80>7 / 8$. Hence, when we allow for general mechanisms, the seller will no longer refrain from inducing the buyer to reveal his type. Random renting contracts allow the seller to induce the separation of types at a lower cost.

\section{The mechanism design problem}

Due to the dynamic nature of our trade setting, the seller may update his belief about the buyer's type from the observation of the buyer's past behavior. Since the buyer 
adjusts his behavior accordingly, the design of the optimal mechanism requires the seller to take account of the mechanism's effect on information revelation. This distinguishes the dynamic setting from a static framework in which seller and buyer interact only once.

Bester and Strausz (2001) have shown that, in a dynamic setting the revelation principle does not restrain the buyer to reveal his true type truthfully but only requires that he does so with a strictly positive probability. We denote by $q_{L} \in[0,1)$ and $q_{H} \in(0,1]$ the probabilities with which the high type and the low type report the message $m=h$ respectively. If the seller induces the buyer to use the reporting strategy $\left(q_{L}, q_{H}\right)$ by offering the menu $\left\{\left(x_{l}, t_{l}\right),\left(x_{h}, t_{h}\right)\right\}$ his updated beliefs conditional on the buyer having reported message $h$ or $l$ are given by

$$
\beta_{h} \equiv \frac{\beta q_{H}}{\beta q_{H}+(1-\beta) q_{L}} \quad \text { and } \quad \beta_{l} \equiv \frac{\beta\left(1-q_{H}\right)}{1-\beta q_{H}-(1-\beta) q_{L}} .
$$

Note that since messages can be renamed we can assume without loss of generality that $\beta_{h} \geq \beta \geq \beta_{l}$ or equivalently $q_{H} \geq q_{L}$. In a separating mechanism both types of buyer report their type truthfully $\left(q_{L}=0, q_{H}=1\right)$, allowing the seller to determine with certainty whether the buyer has a high or a low valuation. In contrast, in a pooling mechanism, $q_{L}=q_{H}$, and the seller's updated belief remains equal to his prior. Finally, a semi-separating mechanism elicits some but not all of the buyer's information, inducing posterior beliefs $\left(\beta_{l}, \beta_{h}\right) \notin\{(0,1),(\beta, \beta)\}$.

In the following we denote by $V_{K}\left(\beta_{m}\right), U_{K}^{H}\left(\beta_{m}\right)$, and $U_{K}^{L}\left(\beta_{m}\right)$ the seller's and the buyer's continuation values when there are $K$ periods of trade remaining and the seller's belief about the buyer's type is given by $\beta_{m}$.

Consider the renting problem when there are $2 \leq K \leq N$ periods of trade remaining and the seller's belief is $\beta \in(0,1)$. Given that we can write the buyer's (ex ante) likelihood to report message $h$ as $\beta q_{H}+(1-\beta) q_{L}=\frac{\beta-\beta_{l}}{\beta_{h}-\beta_{l}}$ the optimal renting mechanism must solve the following 


\section{Renting Program:}

$$
\begin{array}{r}
\max _{x_{l}, x_{h}, t_{l}, t_{h}, \beta_{l}, \beta_{h}} \frac{\beta-\beta_{l}}{\beta_{h}-\beta_{l}}\left[t_{h}+\delta_{S} V_{K-1}\left(\beta_{h}\right)\right]+\frac{\beta_{h}-\beta}{\beta_{h}-\beta_{l}}\left[t_{l}+\delta_{S} V_{K-1}\left(\beta_{l}\right)\right] \\
\text { subject to } \\
x_{h}-t_{h}+\delta_{B} U_{K-1}^{H}\left(\beta_{h}\right) \geq x_{l}-t_{l}+\delta_{B} U_{K-1}^{H}\left(\beta_{l}\right) \\
\theta_{L} x_{l}-t_{l}+\delta_{B} U_{K-1}^{L}\left(\beta_{l}\right) \geq \theta_{L} x_{h}-t_{h}+\delta_{B} U_{K-1}^{L}\left(\beta_{h}\right) \\
x_{h}-t_{h}+\delta_{B} U_{K-1}^{H}\left(\beta_{h}\right) \geq 0 \\
\theta_{L} x_{l}-t_{l}+\delta_{B} U_{K-1}^{L}\left(\beta_{l}\right) \geq 0 .
\end{array}
$$

The constraints (4) and (5) are the dynamic incentive constraints of the high and the low type, respectively. These constraints have to hold with equality whenever the seller wishes to induce the buyer to misreport his type with positive probability. That is, (4) must hold with equality when $q_{H}<1 \Leftrightarrow \beta_{L}>0$ and (5) must hold with equality when $q_{L}>0 \Leftrightarrow \beta_{h}<1$. The constraints (6) and (7) are the corresponding participation constraints.

The selling case differs from the renting case in that, upon delivery, trade terminates and the buyer derives utility from the seller's product in all remaining periods. Abbre-

viating notation by letting $\sigma_{K} \equiv \sum_{n=0}^{K-1} \delta_{B}^{n}$ the optimal selling mechanism must solve the following

\section{Selling Program:}

$$
\begin{array}{r}
\max _{x_{l}, x_{h}, t_{l}, t_{h}, \beta_{l}, \beta_{h}} \frac{\beta-\beta_{l}}{\beta_{h}-\beta_{l}}\left[t_{h}+\left(1-x_{h}\right) \delta_{S} V_{K-1}\left(\beta_{h}\right)\right]+\frac{\beta_{h}-\beta}{\beta_{h}-\beta_{l}}\left[t_{l}+\left(1-x_{l}\right) \delta_{S} V_{K-1}\left(\beta_{l}\right)\right] \\
\text { subject to } \\
x_{h} \sigma_{K}-t_{h}+\left(1-x_{h}\right) \delta_{B} U_{K-1}^{H}\left(\beta_{h}\right) \geq x_{l} \sigma_{K}-t_{l}+\left(1-x_{l}\right) \delta_{B} U_{K-1}^{H}\left(\beta_{l}\right) \\
x_{l} \sigma_{K} \theta_{L}-t_{l}+\left(1-x_{l}\right) \delta_{B} U_{K-1}^{L}\left(\beta_{l}\right) \geq x_{h} \sigma_{K} \theta_{L}-t_{h}+\left(1-x_{h}\right) \delta_{B} U_{K-1}^{L}\left(\beta_{h}\right) \\
x_{h} \sigma_{K}-t_{h}+\left(1-x_{h}\right) \delta_{B} U_{K-1}^{H}\left(\beta_{h}\right) \geq 0 \\
x_{l} \sigma_{K} \theta_{L}-t_{l}+\left(1-x_{l}\right) \delta_{B} U_{K-1}^{L}\left(\beta_{l}\right) \geq 0 .
\end{array}
$$


Again, the dynamic incentive constraints (9) and (10) must hold with equality when $q_{H}<1 \Leftrightarrow \beta_{L}>0$ and $q_{L}>0 \Leftrightarrow \beta_{h}<1$, respectively.

The following lemma is obtained via the steps that are commonly employed in the analysis of the corresponding static problems, i.e., it uses the fact that the participation constraint of the high type is redundant whereas the participation constraint of the low type and the incentive constraint of the high type must be binding at the optimum:

Lemma 1. With $K$ periods of trade remaining and the seller's belief given by $\beta \in(0,1)$, the seller's optimal mechanism sets $x_{h}^{*}=1$ and chooses $x_{l}, \beta_{l}, \beta_{h}$ to solve the following

- Reduced Renting Program:

$$
\begin{aligned}
\max _{x_{l}, \beta_{l}, \beta_{h}} \quad & x_{l} \theta_{L}+\frac{\beta_{h}-\beta}{\beta_{h}-\beta_{l}} \delta_{S} V_{K-1}\left(\beta_{l}\right) \\
& +\frac{\beta-\beta_{l}}{\beta_{h}-\beta_{l}}\left\{1-x_{l}-\delta_{B}\left[U_{K-1}^{H}\left(\beta_{l}\right)-U_{K-1}^{H}\left(\beta_{h}\right)\right]+\delta_{S} V_{K-1}\left(\beta_{h}\right)\right\}
\end{aligned}
$$

subject to

$$
x_{l} \leq 1-\frac{\delta_{B}}{\Delta \theta}\left[U_{K-1}^{H}\left(\beta_{l}\right)-U_{K-1}^{H}\left(\beta_{h}\right)\right] \text { with eq. if } \beta_{h}<1 .
$$

- Reduced Selling Program:

$$
\begin{aligned}
\max _{x_{l}, \beta_{l}, \beta_{h}} \quad & x_{l} \theta_{L} \sigma_{K}+\frac{\beta_{h}-\beta}{\beta_{h}-\beta_{l}}\left(1-x_{l}\right) \delta_{S} V_{K-1}\left(\beta_{l}\right) \\
& +\frac{\beta-\beta_{l}}{\beta_{h}-\beta_{l}}\left(1-x_{l}\right)\left[\sigma_{K}-\delta_{B} U_{K-1}^{H}\left(\beta_{l}\right)\right]
\end{aligned}
$$

subject to

$$
x_{l} \leq 1 \quad \text { with eq. if } \beta_{h}<1 \text {. }
$$

Proof: See the Appendix.

Lemma 1 shows that independently of the mode of trade, the seller's optimal mechanism offers the maximum possible allocation $x_{h}=1$ to the high type buyer. This is familiar from the static setting and follows from the fact that an increase in $x_{h}$ raises the seller's objective while simultaneously relaxing the constraints. Trading with the high 
type is beneficial because it creates surplus while simultaneously improving the seller's ability to elicit the buyer's type.

The lemma also reveals an important difference between renting and selling. Note that in the renting case, the dynamic monotonicity constraint (14) is more demanding than in the selling case (16). This difference is due to the ratchet effect. In the renting case, the existence of future information rents for the high type drives a wedge between the incentive compatible allocations requiring them not only to be monotonically increasing in the reported type but to differ from each other by a strictly positive amount. To understand the origin of this constraint, note from (44) and (5) that the transfer difference $t_{h}-t_{l}$ has to be smaller than $x_{h}-x_{l}-\delta_{B}\left[U_{K-1}^{H}\left(\beta_{l}\right)-U_{K-1}^{H}\left(\beta_{h}\right)\right]$ for the high type to prefer $\left(x_{h}, t_{h}\right)$ but larger than $\left(x_{h}-x_{l}\right) \theta_{L}-\delta_{B}\left[U_{K-1}^{L}\left(\beta_{l}\right)-U_{K-1}^{L}\left(\beta_{h}\right)\right]$ for the low type to prefer $\left(x_{l}, t_{l}\right)$. For $U_{K-1}^{H}\left(\beta_{l}\right)-U_{K-1}^{H}\left(\beta_{h}\right)>U_{K-1}^{L}\left(\beta_{l}\right)-U_{K-1}^{L}\left(\beta_{h}\right)=0$ this is possible only when the allocations $x_{h}$ and $x_{l}$ are sufficiently different. Assumption 1 guarantees that $x_{l}, x_{h} \in[0,1]$ can be chosen sufficiently different for (full) separation of types to be achievable. In particular, it follows from $0 \leq U_{K-1}^{H}\left(\beta_{l}\right)-U_{K-1}^{H}\left(\beta_{h}\right) \leq U_{K-1}^{H}(0)-U_{K-1}^{H}(1)=\Delta \theta \sigma_{K-1}$ and Assumption 1 that the RHS of (14) is strictly positive (for details see the proof of Lemma 11).

Lemma 1 states properties of the optimal renting and selling mechanism that hold independently of the specific nature of the corresponding continuation values. With only one period to go, the distinction between renting and selling ceases to exist and from the well-established solution of the static problem we know that continuation values are given by:

$$
\begin{aligned}
V_{1}\left(\beta_{m}\right) & =\max \left\{\theta_{L}, \beta_{m}\right\} \\
U_{1}^{H}\left(\beta_{m}\right) & = \begin{cases}0 & \text { if } \beta_{m}>\theta_{L} \\
0 \text { or } \Delta \theta & \text { if } \beta_{m}=\theta_{L} \\
\Delta \theta & \text { if } \beta_{m}<\theta_{L}\end{cases} \\
U_{1}^{L}\left(\beta_{m}\right) & =0 .
\end{aligned}
$$


In the following section we solve the reduced programs in Lemma 1 using (17) and (18) to obtain a complete characterization of the optimal renting and selling mechanisms for the case where $N=2$. This characterization will serve as the starting point for our inductive analysis of the general case $N \geq 2$ in Section 6 .

\section{The optimal mechanism for $N=2$}

In this section we characterize the optimal renting and selling mechanisms when there are only two potential periods of trade. Our determination of the optimal mechanism in this section is simplified by the insight, shown formally in the proof of Propositions 1 and 2 , that for $N=2$ and $\beta<\theta_{L}$, the seller cannot benefit from the use of a semi-separating mechanism. Semi-separation can be optimal only when it substitutes for the seller's lack of commitment to maintain a high price (which requires the seller to be tough - see Hart and Tirole (1988)) or when it helps to distribute the revelation of the buyer's type across time (which requires at least three periods - see Section 6).

\section{$5.1 \quad$ Renting}

We first state our result and then explain its intuition.

Proposition 1. Let $N=2$. In the renting case, the seller's revenue is maximized by choosing a (first-period) mechanism with the following characteristics:

- Pooling: For $\beta \in\left(0, \beta_{2}^{R}\right]$ the seller posts a rental-price $p^{*}=\theta_{L}$ which is accepted by both types of buyer.

- Separation: For $\beta \in\left(\beta_{2}^{R}, \theta_{L}\right)$ the seller offers a menu consisting of a random-delivery contract $\left(x_{l}^{*}, t_{l}^{*}\right)=\left(1-\delta_{B},\left(1-\delta_{B}\right) \theta_{L}\right)$ and a secure-delivery contract $\left(x_{h}^{*}, t_{h}^{*}\right)=$ $\left(1, \theta_{L}\right)$ inducing the high and the low type to choose secure- and random-delivery respectively. 
The threshold is given by $\beta_{2}^{R} \equiv \frac{\delta_{B} \theta_{L}}{\delta_{S}-\left(\delta_{S}-\delta_{B}\right) \theta_{L}} \in\left(0, \theta_{L}\right)$. If the seller is restricted to priceposting, he will separate in a strictly smaller interval of priors $\left(\beta_{2}^{R P P}, \theta_{L}\right)$ where $\beta_{2}^{R P P}=$ $\frac{\theta_{L}}{1+\left(\delta_{S}-\delta_{B}\right) \Delta \theta} \in\left(\beta_{2}^{R}, \theta_{L}\right)$.

Proof: See the Appendix.

To understand this result, first consider the mechanism that is optimal amongst all separating mechanism. Substitution of $\beta_{l}=0, \beta_{h}=1$, and the one-period continuation values in (17) and (18) simplifies the reduced renting program in Lemma 1 further to:

$$
\max _{x_{l} \in\left[0,1-\delta_{B}\right]} \beta\left[1-x_{l} \Delta \theta\right]+(1-\beta) x_{l} \theta_{L}+\delta_{S} \theta_{L}+\beta\left(\delta_{S}-\delta_{B}\right) \Delta \theta .
$$

Notice that, except for the constraint on $x_{l}$ and the constant part of the objective, this program is identical to the static (one-period) program. As the seller is soft, $\beta<\theta_{L}$, he would therefore like to choose the likelihood of delivery to the low type, $x_{l}$, as high as possible. However, in the dynamic setting, separation of types requires $x_{l}$ not to exceed the threshold $1-\delta_{B}$. Hence, the seller's optimal separating mechanism is random, i.e., $x_{l}=1-\delta_{B} 10$

Comparing the seller's optimized payoff from separating, obtained by substitution of $x_{l}^{*}=1-\delta_{B}$ into (201), with his payoff from pooling, $\left(1+\delta_{S}\right) \theta_{L}$, we find that separation is optimal if and only if

$$
\beta \delta_{S} \Delta \theta>\beta \delta_{B} \Delta \theta+\left\{\theta_{L}-\beta\left[1-x_{l}^{*} \Delta \theta\right]-(1-\beta) x_{l}^{*} \theta_{L}\right\} .
$$

This inequality compares the benefits (LHS) and costs (RHS) from inducing the buyer to reveal his type in period 1 . The benefits originate from the fact that without separation, the seller would charge a low price to a high type in period 2 whereas he can charge a

\footnotetext{
${ }^{10}$ A tough seller, $\beta \geq \theta_{L}$, would choose $x_{l}$ as low as possible by setting $x_{l}=0$. Hence, for a tough seller, a restriction to price posting is innocuous. Heterogeneous discounting adds nothing (qualitatively) new to the standard analysis of the tough seller case contained in Chapter 9 of Bolton and Dewatripont (2005). The only difference is that, with a patient seller, the interval of priors for which the seller chooses separation rather than semi-separation becomes larger. We have confirmed that, in line with our main results, renting leads to more separation than selling also in the tough seller case. Details are available on request.
} 
high price if he knows the buyer's type to be high. The costs consist of the compensation that the high type must receive to give up his future information rent, plus the period 1 misallocation that is necessary to separate types. The term in parentheses is positive since, in the dynamic setting, separation requires the seller to restrict his trade with the low type although his prior would favor pooling if the setting was static. For $\delta_{S}>\delta_{B}$, separation can be optimal in the dynamic context, because the seller's gain from obtaining information about the buyer's type $\left(\delta_{S} \Delta \theta\right)$ is larger than the buyer's loss from revealing this information $\left(\delta_{B} \Delta \theta\right)$. Separation is optimal when the seller's prior is sufficiently close to $\theta_{L}$ because in this case the cost of a suboptimal period 1 allocation becomes negligible.

Finally, note that for $\beta \in\left(\beta_{2}^{R}, \theta_{L}\right)$, the outcome of the mechanism that induces separation optimally cannot be replicated by price-posting. When the seller is restricted to price-posting, he must set $x_{l}=0$ and separation becomes less profitable because it comes at the cost of a larger first-period misallocation. As a consequence, the range of priors for which separation dominates pooling becomes smaller. It is in this sense that allowing the seller to choose more general mechanisms increases his ability to elicit the buyer's type in the renting case.

Proposition 1 contrasts with the view, suggested by the existing literature on dynamic bilateral trade with short-term commitment, that the restriction to price-posting is innocuous. If the seller is more patient than the buyer then there exists a non-empty range of priors for which price-posting ceases to be optimal in the renting case. It is intuitive and follows immediately from $\beta_{2}^{R}$ that this range increases when discounting becomes more heterogeneous.

\section{$5.2 \quad$ Selling}

Again, we first state our result and then explain its intuition.

Proposition 2. Let $N=2$. In the selling case, the seller's optimal (first-period) mechanism consists of price-posting: 
- Pooling: For $\beta \in\left(0, \beta_{2}^{S}\right]$ the seller posts a price $p^{*}=\left(1+\delta_{B}\right) \theta_{L}$ which is accepted by both types of buyer.

- Separation: For $\beta \in\left(\beta_{2}^{S}, \theta_{L}\right)$ the seller posts a price $p^{*}=1+\delta_{B} \theta_{L}$ which is accepted by the high type but rejected by the low type.

The threshold is given by $\beta_{2}^{S} \equiv \frac{1-\left(\delta_{S}-\delta_{B}\right)}{1-\left(\delta_{S}-\delta_{B}\right) \theta_{L}} \theta_{L} \in\left(0, \theta_{L}\right)$.

To understand this result, first consider the possibility that the seller uses a separating mechanisms. Setting $\beta_{l}=0$ and $\beta_{h}=1$ in the reduced selling program in Lemma 1 leaves the seller with the following problem:

$$
\max _{x_{l} \in[0,1]}\left(1-x_{l}\right)\left[\beta\left(1+\delta_{B} \theta_{L}\right)+(1-\beta) \delta_{S} \theta_{L}\right]+\left(1+\delta_{B}\right) x_{l} \theta_{L}
$$

Note that due to the absence of the ratchet effect, the selling-program places no further constraint on the seller's choice of $x_{l}$. Together with the linearity of the seller's program this implies that $x_{l}^{*} \in\{0,1\}$, i.e., price-posting must be optimal. Setting $x_{l}=1$ attains the payoff of a pooling mechanism. Hence it remains to compare the payoff from pooling with the payoff from separating with $x_{l}=0$. Separation is optimal if and only if

$$
\beta \delta_{S} \Delta \theta-\beta\left(\delta_{S}-\delta_{B}\right) \Delta \theta>\beta \delta_{B} \Delta \theta+\left\{\theta_{L}-\beta-(1-\beta)\left(\delta_{S}-\delta_{B}\right) \theta_{L}\right\}
$$

Similar to the renting case, this inequality compares the benefits (LHS) and costs (RHS) of separation. Again, the costs of separation originate from the compensation necessary to induce the high type to reveal his information and from the loss of not serving the low type in period 1 (in parentheses). The benefits of separation are lower than in the renting case, because of the absence of the ratchet effect. Only in the renting case, the seller is able to extract the additional rent $\beta\left(\delta_{S}-\delta_{B}\right) \Delta \theta$ due to his higher level of patience. However, in the selling case, separation also comes at a different cost. The costs of separation are reduced by the fact that the exclusion of the low type from first period trade gives rise to the opportunity of a second period sale. Selling the second-period access to the 
seller's product to the low valuation buyer in period 2 rather than in period 1 creates the additional payoff $\left(\delta_{S}-\delta_{B}\right) \theta_{L}$ when the seller is more patient than the buyer.

Using a selling model with an arbitrary number of periods and a continuum of types, Skreta (2006) shows that price-posting is optimal when the seller and the buyer have equal discount factors. Proposition 2 shows that, in a model with two periods and two types, this insight remains valid when discount factors differ. Heterogeneous discounting only affects the prices charged. In particular, when the seller is more patient than the buyer, then for moderate priors, the seller will separate types by charging a high price in period 1 whereas under homogeneous discounting both types would be served at a low price.

\subsection{Comparison}

It is well known that, in a model with two periods, renting and selling lead to the same outcome when the seller's and the buyer's discount factors are identical (see Chapter 9 in Bolton and Dewatripont (2005)). When the seller is more patient than the buyer, a trivial difference is that renting results in higher revenue than selling. This can be seen in Figure 1 which plots a numerical example comparing the seller's revenue under renting and selling in dependence of the seller's prior. Renting dominates selling because it allows the patient seller to "sell" the second period access to his product in period 2 rather than in period 1 .

Our analysis in this section shows that, besides its effect on the seller's revenue, heterogeneous discounting introduces further, non-trivial differences between renting and selling. The first difference that arises from a simple comparison of Propositions 11 and 2 is that renting may require a more sophisticated, random contract than the simple priceposting characterizing the optimal selling mechanism. The reason for this difference is that in the renting case the existence of future information rents drives a wedge between the incentive compatible first period trades, whereas in the selling case the ratchet effect 


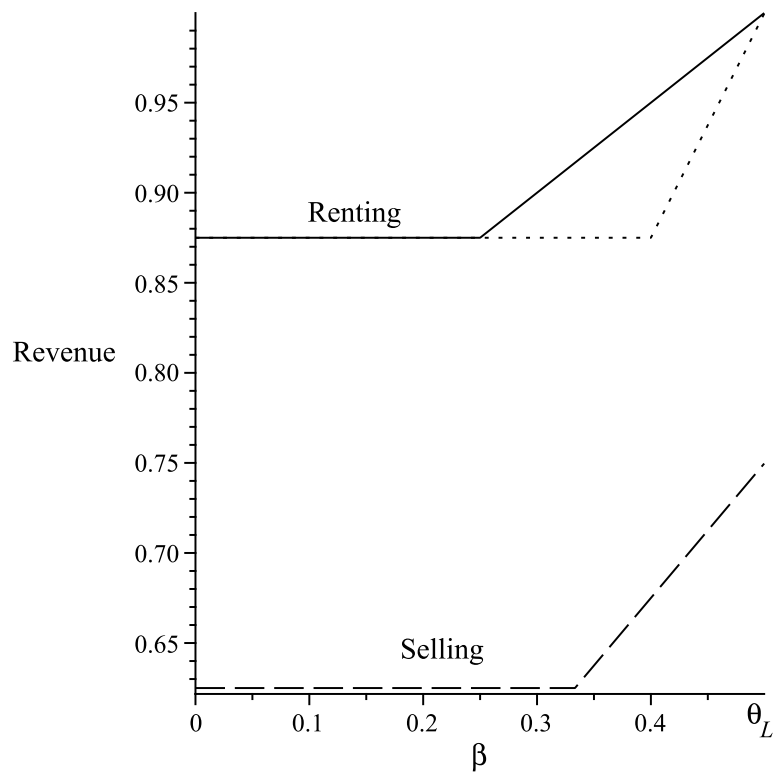

Figure 1: Revenue in Dependence of the Seller's Prior. The figure compares revenue from renting with revenue from selling. In the renting case, revenue is depicted with (dotted) and without (solid) the restriction to price-posting. Note that allowing for general mechanisms can increase the seller's revenue from renting by more than $10 \%$. Parameters are $\theta_{L}=0.5, \delta_{S}=0.75$ and $\delta_{B}=0.25$.

has no bite. This difference matters under heterogeneous but not under homogeneous discounting because a patient seller may prefer separation even when implementing the same trade with both types is optimal from a static viewpoint.

More importantly, Propositions 1 and 2 also allow us to compare renting and selling with respect to the amount of information that becomes revealed. In particular, a comparison of the intervals of priors for which separation occurs gives us the following:

Corollary 1. Let $N=2$. Under renting the seller's optimal mechanism induces more separation than under selling, i.e., $\beta_{2}^{R}<\beta_{2}^{S}$. The opposite holds when the seller is restricted to price-posting, i.e., $\beta_{2}^{R P P}>\beta_{2}^{S}$.

Proof: See the Appendix.

The result is pictured in Figure 2. There we depict the range of prior beliefs for which the optimal mechanism induces the buyer to reveal (completely) his type. The case 


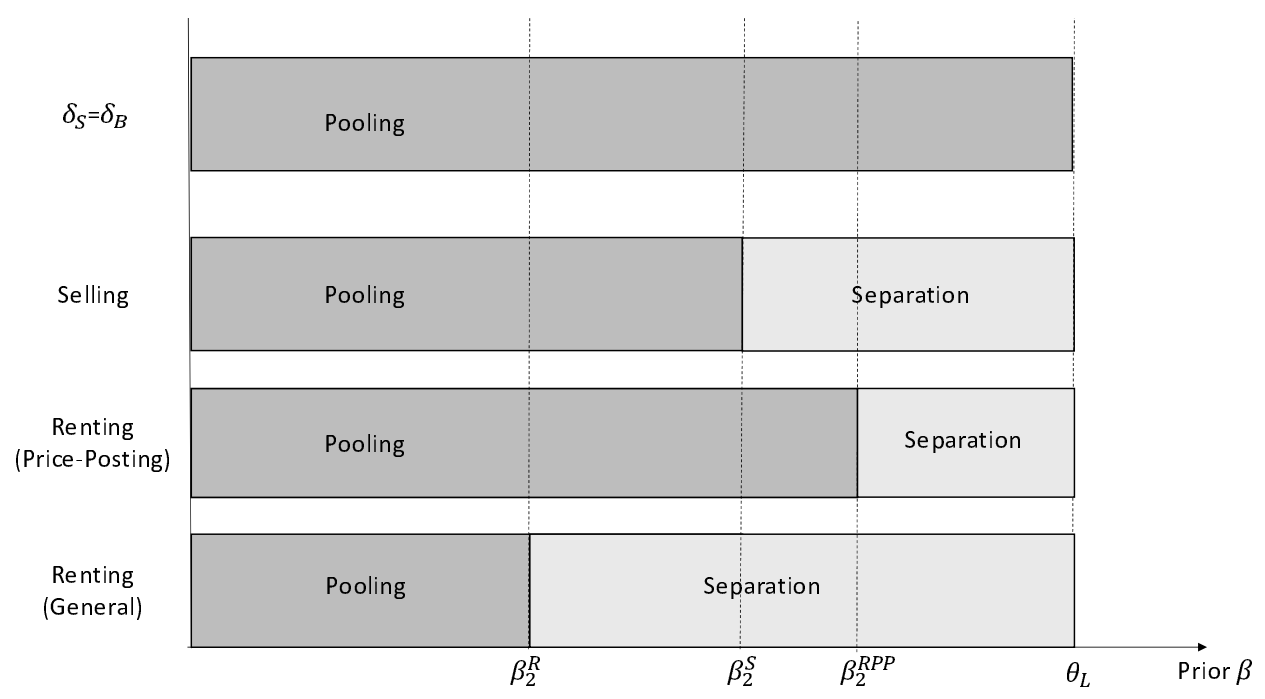

Figure 2: The Optimal Mechanism for $N=2$. The case of homogeneous discounting, $\delta_{S}=\delta_{B}$ serves as a benchmark. When the seller is more patient than the buyer, selling and renting differ with respect to the degree of information revelation, and the comparison depends on whether or not general mechanisms are available.

of homogeneous discounting serves as a benchmark. Under homogeneous discounting, renting and selling are equivalent and the optimal mechanism pools the buyer's types for all priors $\beta<\theta_{L}$. Allowing for heterogeneous discounting shows that, generically, renting and selling are different. Renting leads to more learning than selling but only when general mechanisms are available.

The intuition for Corollary 1 can be obtained from a comparison of the benefits and the costs of separation under the two modes of trade. Consider first the case where the seller is restricted to simple price-posting. Setting $x_{l}=0$ in (21) and comparing with (23) we see that selling reduces the costs of separation by $\left(\delta_{S}-\delta_{B}\right)\left(\theta_{L}-\beta \theta_{L}\right)$ while lowering the benefits of separation by only $\left(\delta_{S}-\delta_{B}\right)\left(\beta-\beta \theta_{L}\right)$. In other words, when the seller is restricted to price posting, the elimination of the ratchet effect through selling rather than renting improves the seller's ability to learn the buyer's type. Allowing the seller to use general mechanisms reverses the cost-benefit comparison in the favor of renting, by lowering the costs of separation in the renting case. With the help of a random-delivery, the seller can achieve separation under renting without excluding the low type buyer from 
first period trade altogether. When this effect is taken into account, the seller's incentive to separate is stronger under renting than under selling.

\section{$6 \quad$ Arbitrary number of periods $N \geq 2$}

The characterization of the optimal mechanisms for the two-period model contained in the previous section serves as the starting point for an inductive analysis of the general case with an arbitrary number of periods. In this section, we show that the two main insights of the two-period case remain valid: With $N \geq 2$ periods of trade, renting involves the use of a random mechanism and leads to less pooling than selling. A new feature of the model with more than two periods is the emergence of a novel kind of semi-separating mechanism. Instead of substituting for the seller's lack of commitment to a high price (well known from Hart and Tirole's (1988) analysis of the tough seller's case), with a soft seller semi-separating mechanisms serve the purpose of distributing/delaying type-revelation across periods and turn out to be optimal for moderate priors.

\subsection{Robustness}

The following two results are the $N$-period analogs of Propositions 1 and 2 , Their proof is by induction over the number of periods and is based on the intuition explained in the previous section.

Proposition 3. In the renting case, there exist thresholds $\beta_{N}^{R}<\beta_{N-1}^{R}<\cdots<\beta_{2}^{R}<\beta_{1}^{R}=$ $\theta_{L}$ such that with $2 \leq K \leq N$ periods of trade remaining and the seller's belief given by $\beta \in\left[0, \theta_{L}\right)$ the following holds:

- Pooling: For $\beta \in\left[0, \beta_{K}^{R}\right]$ the seller posts a rental-price $p^{*}=\theta_{L}$ in all $K$ remaining periods and both types of buyer accept.

- Separation or Semi-Separation: For $\beta \in\left(\beta_{K}^{R}, \beta_{K-1}^{R}\right]$ the seller offers a menu consisting of a random-delivery contract $x_{l} \in(0,1)$ and a secure-delivery contract $x_{h}=1$. 
The high type chooses secure-delivery whereas the low type is induced to choose random-delivery or to mix.

Proposition 4. In the selling case, there exist thresholds $\beta_{N}^{S}<\beta_{N-1}^{S}<\cdots<\beta_{2}^{S}<\beta_{1}^{S}=$ $\theta_{L}$ such that with $2 \leq K \leq N$ periods remaining and the seller's belief given by $\beta \in\left[0, \theta_{L}\right)$, the seller's optimal selling mechanism consists of price-posting:

- Pooling: For $\beta \in\left[0, \beta_{K}^{S}\right]$ the seller posts the price $\theta_{L} \sigma_{K}$ which is accepted by both types of buyer.

- Separation: For $\beta \in\left(\beta_{K}^{S}, \beta_{K-1}^{S}\right]$ the seller posts the price $\sigma_{K}-\delta_{B} \Delta \theta \sigma_{K-1}$ which is accepted by the high type but rejected by the low type. For $\beta>\beta_{K-1}^{S}$ separation might not be optimal but dominates pooling.

Besides the potential emergence of semi-separation in the renting case (discussed below), Propositions 3 and 4 differ from the two-period case in that they only provide a partial characterization of the seller's optimal mechanism. In particular, with $K$ periods to go, the optimal mechanism is determined only for a subset $\left(0, \beta_{K-1}\right)$ of the seller's priors. However, the fact that for $K=1,2, \ldots, N$ these subsets cover the entire range of priors $\left[0, \theta_{L}\right)$ allows us to obtain the following result about a (soft) seller's propensity to elicit the buyer's type in a dynamic trade-setting:

Corollary 2. Consider the range of priors $\left[0, \beta_{N}\right]$ for which the seller pools both types of buyer during all $N$ periods of trade. Allowing for mechanisms more general than priceposting reduces pooling (only) in the renting case, i.e., $\left[0, \beta_{N}^{R}\right] \subset\left[0, \beta_{N}^{R P P}\right]$. With an infinite horizon, renting leads to less pooling than selling, i.e., $\left[0, \beta_{\infty}^{R}\right] \subset\left[0, \beta_{\infty}^{S}\right]$. If $\delta_{S} \in$ $\left(\delta_{B}, \frac{1-\delta_{B}}{1-\delta_{B}^{N-1}}\right]$ then $\left[0, \beta_{N}^{R}\right] \subset\left[0, \beta_{N}^{S}\right]$ independently of $N$.

The corollary's first claim is immediate from Proposition 3 which shows that, in the renting case, the seller's optimal separating mechanism involves a random allocation. A restriction to price-posting therefore decreases the seller's payoffs from separation, making 
pooling relatively more attractive. In the selling case a restriction to price-posting has no effect because, as shown by Proposition 4, price-posting constitutes the seller's optimal way to separate the buyer's types.

To understand why, in the renting case, $\beta_{N}^{R}$ serves as the boundary of the pooling range, note from Propositions 3 that for $\beta \in\left[0, \beta_{N}^{R}\right]$ pooling in all $N$ periods is optimal for the seller. For $\beta \notin\left[0, \beta_{N}^{R}\right]$, there exists a $K \leq N$ such that after pooling for $N-K$ periods, separation or semi-separation becomes optimal (because the number of remaining periods has decreased from $N$ to $K$ whereas $\beta$ has remained unchanged). Hence for $\beta \notin\left[0, \beta_{N}^{R}\right]$, pooling for all $N$ periods cannot be optimal.

Corollary 2 complements Corollary 1 in showing that renting leads to more informationrevelation than selling (in the sense defined in the corollary), not only with a short $(N=2)$ but also with a long $(N=\infty)$ horizon. The comparison between renting and selling for a general $N$ is complicated by the fact that for $N>2$ we can only determine an upper bound on the threshold $\beta_{N}^{R}$ (see proof of Corollary 21). However, under the additional assumption that the seller is not too patient, we are able to show that renting leads to less pooling than selling independently of the length of the horizon $N 11$ The difference with respect to the two period model, is that for general $N$, random mechanisms, although optimal, may cease to be necessary for our conclusion that renting leads to more information-revelation than selling. This happens when the time horizon is sufficiently long. In particular, for an infinite horizon we find that renting leads to less pooling than selling even under the restriction to price-posting, i.e., $\left[0, \beta_{\infty}^{R P P}\right] \subset\left[0, \beta_{\infty}^{S}\right]$.

\subsection{Novel features}

Our result that renting leads to less pooling than selling comes at a surprise in light of the existing literature. Hart and Tirole's (1988) Proposition 3 has shown that in a renting

\footnotetext{
${ }^{11}$ Assumption 11 guarantees that the set of discount factors $\left(\delta_{B}, \frac{1-\delta_{B}}{1-\delta_{B}^{N-1}}\right]$ contained in Corollary 2 is non-empty.
} 
framework with a tough seller, homogeneous discounting, and price-posting, the buyer's information may become revealed only during the final periods of trade. Their result relies on the assumption that the buyer's discount factor is greater than one half, which guarantees that, with a sufficient number of periods remaining, the seller is unable to induce the high type to reveal his information by accepting a price above the low type's valuation (see our explanation of dynamic incentive compatibility following Lemma 1). In our setting, the buyer's discount factor has been assumed to be small enough for such separation to be feasible right from the start. In spite of our finding that, when feasible, separation turns out to be optimal for a larger range of priors than in the selling case, there exists a sense in which Hart and Tirole's insight about renting being cursed by "delayed type-revelation" remains valid. In the following we consider a simple three-period example to highlight this point.

Let $N=3$ and consider a renting mechanism which offers the menu

$$
\left(x_{l}, t_{l}\right)=\left(1-\delta_{B}^{2},\left(1-\delta_{B}^{2}\right) \theta_{L}\right) \text { and }\left(x_{h}, t_{h}\right)=\left(1, \theta_{L}\right)
$$

inducing the posteriors $\beta_{l}=0$ and $\beta_{h}=\theta_{L}$. Using Proposition 1 to calculate the corresponding continuation payoffs $V_{2}\left(\beta_{m}\right)$ and $U_{2}^{H}\left(\beta_{m}\right)$, the seller's payoff from using this semi-separating mechanism is readily determined from (13):

$$
V^{s e m i}=\theta_{L}+\delta_{S} \theta_{L}+\left(\delta_{S}^{2}-\delta_{B}^{2}\right) \theta_{L}+\beta\left[\delta_{B}^{2}+\delta_{S}\left(\delta_{S}-\delta_{B}\right) \Delta \theta\right]
$$

Comparison with the payoffs from pooling, $V^{\text {pool }}=\theta_{L}\left(1+\delta_{S}+\delta_{S}^{2}\right)$, and separation, $V^{\text {sep }}=\theta_{L}+(1-\beta) \theta_{L}\left(\delta_{S}-\delta_{B}+\delta_{S}^{2}-\delta_{B}^{2}\right)+\beta\left(\delta_{S}+\delta_{S}^{2}\right)$, reveals that this semi-separating mechanism dominates pooling and separation when

$$
\beta>\frac{\delta_{B}^{2} \theta_{L}}{\delta_{B}^{2}+\delta_{S}\left(\delta_{S}-\delta_{B}\right) \Delta \theta} \quad \text { and } \quad \beta<\frac{\delta_{B} \theta_{L}}{\left(1+\delta_{B}\right)\left[\delta_{S}-\left(\delta_{S}-\delta_{B}\right) \theta_{L}\right]-\delta_{B}^{2}}
$$

respectively. There exist priors $\beta$ that satisfy both of these inequalities when $\delta_{S}>\delta_{B}(1+$ $\left.\delta_{B}\right)$. Semi-separation constitutes a "compromise" between pooling and (full) separation 
which turns out to be optimal for moderate values of the seller's prior 12 It induces the buyer to reveal his type only partially, before becoming fully separated in the next period. The seller delays the revelation of the buyer's type because his prior is too optimistic to pool but not optimistic enough to immediately separate the buyer. With a soft seller, semi-separation serves as a means to distribute information-rents across trading-periods. This distinguishes the above mechanism from the semi-separating mechanisms commonly found to be optimal in models with a tough seller. A tough seller restricts his learning in order to maintain tough, whereas a soft seller restricts his learning to prevent himself from becoming tough too early. While the former requires $\beta_{l}>0$ or equivalently $q_{H}<1$, for the latter it has to hold that $\beta_{h}<1$ or equivalently $q_{L}>0$. In other words, with a tough seller, semi-separation induces type-misrepresentation by the high type whereas with a soft seller, it is the low type who is allowed to misrepresent. 13 These features of the seller's optimal mechanism are non-existent for $N=2$ because with only two periods of trade all (potential) learning is restricted to a single occasion.

\section{Conclusion}

In this paper we have applied a mechanism design approach to a model of dynamic bilateral trade with short-term commitment. Our analysis encompasses both the renting and the selling case. Allowing the seller to be more patient than the buyer reveals two differences between these alternative modes of trade which become overlooked under the simplifying assumption of homogeneous discounting. First, the optimal renting mechanism involves a menu containing a secure- and a random-delivery contract whereas the outcome of the optimal selling mechanism can be implemented via simple price-posting.

\footnotetext{
${ }^{12}$ Proving optimality of the mechanism in (24) requires further comparisons with alternative semiseparating mechanisms. We have confirmed that there exist parameters for which the mechanism in (24) constitutes the seller's optimal mechanism.

${ }^{13}$ In our semi-separating mechanism, the seller becomes progressively optimistic about the buyer's type or learns that his type is low. This contrasts with the sequential separating dynamics of Maestri's (2017) renegotiation equilibria where the seller's posterior about the buyer's type decreases unless he finds out that the buyer's type is high.
} 
Second, renting induces the buyer to reveal his type for a wider range or parameters than selling. Both results contrast with the view suggested by the existing literature which holds that the restriction to price-posting is innocuous and that informational asymmetries are more persistent under renting than under selling. The fact that our second result relies on the availability of a random-delivery contract underlines the importance of a general mechanism design approach for models of dynamic adverse selection.

\section{Acknowledgements}

We thank V. Bhaskar, Wouter Dessein, Mikhail Drugov, Dino Gerardi, Angel Hernando Veciana, Daniel Krähmer, Antoine Loeper, Margaret Meyer, Konrad Mierendorff, and Roland Strausz for valuable discussions and suggestions.

\section{Appendix - Proofs}

Proof of Lemma 1: This proof has three steps. Only the last step requires the distinction between renting and selling. (i) The participation constraint for the high type is redundant: As $\theta_{L}<\theta_{H}=1$ and $U_{K-1}^{H}\left(\beta_{l}\right) \geq U_{K-1}^{L}\left(\beta_{l}\right)$, (4) and (7) together imply (6), and (9) and (12) together imply (11) . (ii) The participation constraint of the low type and the incentive constraint of the high type are binding at the optimum: If (17) or (12) holds with strict inequality then there exists an $\epsilon>0$ such that raising both transfers to $t_{l}+\epsilon$ and $t_{h}+\epsilon$ increases the seller's revenue while keeping all constraints satisfied. If (4) or (9) holds with strict inequality then there exists an $\epsilon>0$ such that raising the transfer for the buyer reporting a high type to $t_{h}+\epsilon$ increases the seller's revenue while keeping all constraints satisfied. (iii) To show that $x_{h}^{*}=1$ consider renting and selling in separation:

Renting: As (4) and (7) are binding we can substitute $t_{l}^{*}=x_{l} \theta_{L}+\delta_{B} U_{K-1}^{L}\left(\beta_{l}\right)$ and 
$t_{h}^{*}=t_{l}^{*}+x_{h}-x_{l}+\delta_{B}\left[U_{K-1}^{H}\left(\beta_{h}\right)-U_{K-1}^{H}\left(\beta_{l}\right)\right]$ into (8) to make the objective equal to

$$
\begin{array}{r}
\theta_{L} x_{l}+\delta_{B} U_{K-1}^{L}\left(\beta_{l}\right)+\frac{\beta_{h}-\beta}{\beta_{h}-\beta_{l}} \delta_{S} V_{K-1}\left(\beta_{l}\right) \\
+\frac{\beta-\beta_{l}}{\beta_{h}-\beta_{l}}\left\{x_{h}-x_{l}+\delta_{B}\left[U_{K-1}^{H}\left(\beta_{h}\right)-U_{K-1}^{H}\left(\beta_{l}\right)\right]+\delta_{S} V_{K-1}\left(\beta_{h}\right)\right\}
\end{array}
$$

Substitution of $t_{l}^{*}$ and $t_{h}^{*}$ into the remaining constraint (5) gives

$$
\left(x_{h}-x_{l}\right) \frac{\Delta \theta}{\delta_{B}} \geq\left[U_{K-1}^{H}\left(\beta_{l}\right)-U_{K-1}^{H}\left(\beta_{h}\right)\right]-\left[U_{K-1}^{L}\left(\beta_{l}\right)-U_{K-1}^{L}\left(\beta_{h}\right)\right] .
$$

Because an increase in $x_{h}$ raises the objective in (27) while relaxing the constraint in (28), setting $x_{h}^{*}=1$ must maximize the seller's payoff. Substitution of $x_{h}=1$ and $U_{K-1}^{L}=0$ (which holds because, as shown above, the participation constraint of the low type must be binding in every period) into (27) and (28) leads to the reduced renting program.

Note that the constraint (14) together with the fact that $x_{l} \geq 0$ requires that $U_{K-1}^{H}\left(\beta_{l}\right)-$ $U_{K-1}^{H}\left(\beta_{h}\right) \leq \frac{\Delta \theta}{\delta_{B}}$. This means that if $U_{K-1}^{H}(0)-U_{K-1}^{H}(1)>\frac{\Delta \theta}{\delta_{B}} \Leftrightarrow \sum_{n=1}^{K} \delta_{B}^{n}>1$ then there exists an upper bound on the degree of separation that can be induced. Our assumption that $\sum_{n=1}^{N} \delta_{B}^{n}<1$ implies that no such bound exist for any $K \leq N$, i.e., full separation is always feasible. Intuitively, $\delta_{B}$ is assumed to be sufficiently small, such that the (high type) buyer can be induced to give up his future information rent via the threat of a decrease in the current period's probability of delivery from $x_{h}=1$ to $x_{l} \in(0,1)$.

Selling: As (9) and (12) are binding we can substitute $t_{l}^{*}=x_{l} \sigma_{K} \theta_{L}+\left(1-x_{l}\right) \delta_{B} U_{K-1}^{L}\left(\beta_{l}\right)$ and $t_{h}^{*}=t_{l}^{*}+\left(x_{h}-x_{l}\right) \sigma_{K}+\left(1-x_{h}\right) \delta_{B} U_{K-1}^{H}\left(\beta_{h}\right)-\left(1-x_{l}\right) \delta_{B} U_{K-1}^{H}\left(\beta_{l}\right)$ into (8) to make the objective equal to

$$
\begin{array}{r}
\theta_{L} x_{l} \sigma_{K}+\left(1-x_{l}\right) \delta_{B} U_{K-1}^{L}\left(\beta_{l}\right)+\frac{\beta_{h}-\beta}{\beta_{h}-\beta_{l}}\left(1-x_{l}\right) \delta_{S} V_{K-1}\left(\beta_{l}\right)(29) \\
+\frac{\beta-\beta_{l}}{\beta_{h}-\beta_{l}}\left\{\left(x_{h}-x_{l}\right) \sigma_{K}+\left(1-x_{h}\right)\left[\delta_{B} U_{K-1}^{H}\left(\beta_{h}\right)+\delta_{S} V_{K-1}\left(\beta_{h}\right)\right]-\left(1-x_{l}\right) \delta_{B} U_{K-1}^{H}\left(\beta_{l}\right)\right\} .
\end{array}
$$

As $\delta_{B} U_{K-1}^{H}\left(\beta_{h}\right)+\delta_{S} V_{K-1}\left(\beta_{h}\right)<\sigma_{K}$ the modified objective is increasing in $x_{h}$. Substitution of $t_{l}^{*}$ and $t_{h}^{*}$ into the remaining constraint (10) gives

$$
\left(x_{h}-x_{l}\right) \frac{\Delta \theta \sigma_{K}}{\delta_{B}} \geq\left(1-x_{l}\right)\left[U_{K-1}^{H}\left(\beta_{l}\right)-U_{K-1}^{L}\left(\beta_{l}\right)\right]-\left(1-x_{h}\right)\left[U_{K-1}^{H}\left(\beta_{h}\right)-U_{K-1}^{L}\left(\beta_{h}\right)\right] .
$$


As $\delta_{B}\left[U_{K-1}^{H}\left(\beta_{h}\right)-U_{K-1}^{L}\left(\beta_{h}\right)\right]<\Delta \theta \sigma_{K}$, an increase in $x_{h}$ relaxes this constraint. Because an increase in $x_{h}$ raises the objective while relaxing the constraint, setting $x_{h}^{*}=1$ must be optimal. Substituting $x_{h}^{*}=1$ and $U_{K-1}^{L}=0$ into (29) gives the objective in (15). Moreover, for $x_{h}^{*}=1$, (30) is always satisfied and holds with equality if and only if $x_{l}=1$. Hence, in order to induce $\beta_{h}<1$ the seller has to choose $x_{l}=1$.

Proof of Propositions 1 and 2; The comparison of the payoffs from pooling $\left(\beta_{l}=\right.$ $\left.\beta_{h}=\beta\right)$ and separation $\left(\beta_{l}=0, \beta_{h}=1\right)$ is contained in the main text. It remains to show that no mechanism can give the seller a higher revenue than what he can achieve by either pooling or separating:

Renting: Consider a mechanism for which $\beta_{h} \in\left(\theta_{L}, 1\right)$. Continuation values are then $U_{1}^{H}\left(\beta_{l}\right)=\Delta \theta, U_{1}^{H}\left(\beta_{h}\right)=0, V_{1}\left(\beta_{h}\right)=\beta_{h}$, and $V_{1}\left(\beta_{l}\right)=\theta_{L}$. Moreover, (14) must be binding and we can substitute $x_{l}=1-\delta_{B}$ into the reduced renting program (13) which leaves us with the simplified problem:

$$
\max _{\beta_{l} \in[0, \beta], \beta_{h} \in\left(\theta_{L}, 1\right)}\left(1-\delta_{B}\right) \theta_{L}+\frac{\beta_{h}-\beta}{\beta_{h}-\beta_{l}} \delta_{S} \theta_{L}+\frac{\beta-\beta_{l}}{\beta_{h}-\beta_{l}}\left(\delta_{B} \theta_{L}+\delta_{S} \beta_{h}\right) .
$$

Since (31) is decreasing in $\beta_{l}$ for all $\beta_{H}$, setting $\beta_{l}=0$ must be optimal. The resulting payoff $\theta_{L}\left[1+\left(\delta_{S}-\delta_{B}\right)\left(1-\frac{\beta}{\beta_{h}}\right)\right]+\beta \delta_{S}$ is increasing in $\beta_{h}$, i.e., letting $\beta_{h} \rightarrow 1$ must be optimal. Now consider a mechanism with $\beta_{h} \leq \theta_{L}$. Continuation values are $U_{1}^{H}\left(\beta_{l}\right)=$ $U_{1}^{H}\left(\beta_{h}\right)=\Delta \theta$, and $V_{1}\left(\beta_{h}\right)=V_{1}\left(\beta_{l}\right)=\theta_{L} 14$ As (14) must be binding, $x_{l}=1$, the seller's revenue becomes equal to $\left(1+\delta_{S}\right) \theta_{L}$ independently of the induced beliefs, i.e., setting $\beta_{l}=\beta_{h}=\beta$ must be optimal. We have therefore shown that no renting mechanism can give the seller a higher payoff than what he gets by either separating or pooling.

Selling: For $\beta_{h}<1$, the constraint (16) of the reduced selling program must bind and the objective (15) becomes equal to the pooling payoff $\left(1+\delta_{B}\right) \theta_{L}$. For $\beta_{h}=1$, the linearity of the seller's program in $x_{l}$ implies that $x_{l} \in\{0,1\}$. Setting $x_{l}=1$ again leads

\footnotetext{
${ }^{14}$ For $\beta_{h}=\theta_{L}$ the seller is indifferent between pooling and separating in period 2. Hence he can credibly promise to pool in period 2 , i.e., $U_{1}^{H}\left(\beta_{h}\right)=\Delta \theta$, in order to reduce the buyer's reluctance to reveal his type.
} 
the pooling payoff. For $x_{l}=0$ it remains to solve

$$
\max _{\beta_{l} \in[0, \beta]} \frac{\beta-\beta_{l}}{1-\beta_{l}}\left(1+\delta_{B} \theta_{L}\right)+\frac{1-\beta}{1-\beta_{l}} \delta_{S} \theta_{L}
$$

where we have used the fact that $\beta_{l} \leq \beta<\theta_{L}$ and hence $U_{1}^{H}\left(\beta_{l}\right)=\Delta \theta$ and $V_{1}\left(\beta_{l}\right)=\theta_{L}$. As the objective in (32) is decreasing in $\beta_{l}$, setting $\beta_{l}=0$ is optimal. We have therefore shown that no selling mechanism can give the seller a higher payoff than what he gets by either separating or pooling.

Proof of Corollary 1: Note that $\beta_{2}^{R}<\beta_{2}^{S}$ if and only if

$$
\delta_{B}\left[1-\left(\delta_{S}-\delta_{B}\right) \theta_{L}\right]<\left[1-\left(\delta_{S}-\delta_{B}\right)\right]\left[\delta_{S}-\left(\delta_{S}-\delta_{B}\right) \theta_{L}\right] \Leftrightarrow 0<\left(1-\delta_{S}\right) \Delta \theta
$$

Also note that $\beta_{2}^{R P P}>\beta_{2}^{S}$ if and only if

$$
\Leftrightarrow 1-\left(\delta_{S}-\delta_{B}\right) \theta_{L}>\left[1-\left(\delta_{S}-\delta_{B}\right)\right]\left[1+\left(\delta_{S}-\delta_{B}\right) \Delta \theta\right] \Leftrightarrow 0>-\left(\delta_{S}-\delta_{B}\right)^{2} \Delta \theta
$$

This proves the comparative statics contained in Corollary 1 .

Proof of Proposition 3: By induction over the number of periods $N$. Our analysis of the two-period model has shown that for $N=2$ the seller's optimal mechanism satisfies the properties described in Proposition 3. Assume that Proposition 3 holds for $N-1$ and consider the seller's choice of mechanism when there are $N$ period and the seller's belief $\beta$ is such that $\beta<\beta_{N-1}^{R}$. In analogy to $\sigma_{N}=\sum_{n=0}^{N-1} \delta_{B}^{n}$ define $\sigma_{N}^{S} \equiv \sum_{n=0}^{N-1} \delta_{S}^{n}$.

Starting point is the reduced renting program in Lemma 1 with $K=N$. First note that for $\beta_{h}=1$ the objective is (linearly) increasing in $x_{l}$ if and only if

$$
-\frac{\beta-\beta_{l}}{1-\beta_{l}} \Delta \theta+\left(1-\frac{\beta-\beta_{l}}{1-\beta_{l}}\right) \theta_{L}>0 \Leftrightarrow \beta_{l}>\frac{\beta-\theta_{L}}{\Delta \theta}
$$

which is satisfied for all $\beta_{l} \in[0, \beta]$ because $\beta<\beta_{N-1}^{R}<\theta_{L}$. Hence, the constraint must be binding not only for $\beta_{h}<1$ but also for $\beta_{h}=1$ and we can substitute $x_{l}$ into the objective to obtain the simplified program:

$$
\begin{aligned}
& \max _{\beta_{h}, \beta_{l}} \theta_{L}+ \delta_{S} V_{N-1}\left(\beta_{l}\right)+\frac{\beta-\beta_{l}}{\beta_{h}-\beta_{l}} \delta_{S}\left[V_{N-1}\left(\beta_{h}\right)-V_{N-1}\left(\beta_{l}\right)\right] \\
&+\left(1-\frac{\beta-\beta_{l}}{\beta_{h}-\beta_{l}}\right) \delta_{B} \frac{\theta_{L}}{\Delta \theta}\left[U_{N-1}^{H}\left(\beta_{h}\right)-U_{N-1}^{H}\left(\beta_{l}\right)\right] .
\end{aligned}
$$


Using our induction assumption we know that $V_{N-1}\left(\beta_{l}\right)=V_{N-1}\left(\beta_{h}\right)=V_{N-1}(0)=\theta_{L} \sigma_{N-1}^{S}$ and $U_{N-1}^{H}\left(\beta_{l}\right)=U_{N-1}^{H}\left(\beta_{h}\right)=U_{N-1}^{H}(0)=\Delta \theta \sigma_{N-1}$ for all $\left(\beta_{l}, \beta_{h}\right)$ such that $\beta_{l} \leq \beta_{h} \leq \beta_{N-1}^{R}$. In all these cases, the seller's payoff is identical to the one he can obtain by pooling via the choice of $\beta_{l}=\beta_{h}=\beta$ :

$$
V_{N}^{p o o l}=\theta_{L}+\delta_{S} V_{N-1}(0)=\theta_{L} \sigma_{N}^{S}
$$

Alternatively, the seller can separate the buyer's types by choosing a $\left(\beta_{l}, \beta_{h}\right)$ such that $\beta_{l}<\beta<\beta_{N-1}^{R}<\beta_{h}$. Because for all such $\left(\beta_{l}, \beta_{h}\right)$ it holds that $V_{N-1}\left(\beta_{l}\right)=\theta_{L} \sigma_{N-1}^{S}$ and $U_{N-1}^{H}\left(\beta_{h}\right)-U_{N-1}^{H}\left(\beta_{l}\right)=U_{N-1}^{H}\left(\beta_{h}\right)-\Delta \theta \sigma_{N-1} \leq 0$, this can be optimal only if $V_{N-1}\left(\beta_{h}\right)-$ $V_{N-1}\left(\beta_{l}\right)=V_{N-1}\left(\beta_{h}\right)-\theta_{L} \sigma_{N-1}^{S}>0$. Moreover, because $\beta_{l}$ affects the seller's program only through its negative influence on $\frac{\beta-\beta_{l}}{\beta_{h}-\beta_{l}}$, it follows that if the seller chooses to separate, then he must do so optimally with $\beta_{l}=0$. Hence we can define

$$
\beta_{h}^{*} \equiv \arg \max _{\beta_{h} \in\left(\beta_{N-1}^{R}, 1\right]} \frac{\beta}{\beta_{h}} \delta_{S}\left[V_{N-1}\left(\beta_{h}\right)-\theta_{L} \sigma_{N-1}^{S}\right]+\left(1-\frac{\beta}{\beta_{h}}\right) \delta_{B} \frac{\theta_{L}}{\Delta \theta}\left[U_{N-1}^{H}\left(\beta_{h}\right)-\Delta \theta \sigma_{N-1}\right]
$$

and conclude that if the seller chooses to separate with $N$ periods to go then he must do so optimally with $\left(\beta_{l}, \beta_{h}\right)=\left(0, \beta_{h}^{*}\right)$ and $x_{l}^{*}=1-\delta_{B} \sigma_{N-1}+\delta_{B} \frac{U_{N-1}^{H}\left(\beta_{h}^{*}\right)}{\Delta \theta} \in(0,1)$, resulting in the payoff

$$
V_{N}^{s e p}=V_{N}^{\text {pool }}+\frac{\beta}{\beta_{h}^{*}} \delta_{S}\left[V_{N-1}\left(\beta_{h}^{*}\right)-\theta_{L} \sigma_{N-1}^{S}\right]+\left(1-\frac{\beta}{\beta_{h}^{*}}\right) \delta_{B} \frac{\theta_{L}}{\Delta \theta}\left[U_{N-1}^{H}\left(\beta_{h}^{*}\right)-\Delta \theta \sigma_{N-1}\right] .
$$

Define $f(\beta) \equiv V_{N}^{s e p}-V_{N}^{\text {pool }}$. (1) $f$ is continuous in $\beta$. (2) $f(0)=\delta_{B} \frac{\theta_{L}}{\Delta \theta}\left[U_{N-1}\left(\beta_{h}^{*}\right)-\right.$ $\left.\Delta \theta \sigma_{N-1}\right]<0$, because from above we know that $V_{N-1}\left(\beta_{h}^{*}\right)>\theta_{L} \sigma_{N-1}^{S}$ which implies that $U_{N-1}^{H}\left(\beta_{h}^{*}\right)<\Delta \theta \sigma_{N-1}$ (the seller can only gain from separation when the high type buyer looses from it). (3) For the same reason, $f$ is strictly increasing in $\beta$. Finally, (4) $f\left(\beta_{N-1}^{R}\right)>0$. To prove this last property, note that, by our induction assumption, for $\beta=\beta_{N-1}^{R}$ and with $N-1$ periods to go, the seller would be indifferent between pooling forever and separating with some $\left(\beta_{l}, \beta_{h}\right)=\left(0, \tilde{\beta}_{h}\right)$. Hence

$$
\beta_{N-1}^{R}=\tilde{\beta}_{h} \frac{\delta_{B} \frac{\theta_{L}}{\Delta \theta}\left[U_{N-2}^{H}(0)-U_{N-2}^{H}\left(\tilde{\beta}_{h}\right)\right]}{\delta_{S}\left[V_{N-2}\left(\tilde{\beta}_{h}\right)-V_{N-2}(0)\right]+\delta_{B} \frac{\theta_{L}}{\Delta \theta}\left[U_{N-2}^{H}(0)-U_{N-2}^{H}\left(\tilde{\beta}_{h}\right)\right]} .
$$


Suppose that for $\beta=\beta_{N-1}^{R}$ and with $N$ periods to go, the seller separates by inducing the same posteriors $\left(\beta_{l}, \beta_{h}\right)=\left(0, \tilde{\beta}_{h}\right)$. By definition of $\beta_{h}^{*}$, the seller's payoff from separating with $\left(\beta_{l}, \beta_{h}\right)=\left(0, \tilde{\beta}_{h}\right)$ must be (weakly) less than his payoff from separating with $\left(\beta_{l}, \beta_{h}\right)=$ $\left(0, \beta_{h}^{*}\right)$. Hence, $f\left(\beta_{N-1}^{R}\right)>0$ if

$$
\frac{\beta_{N-1}^{R}}{\tilde{\beta}_{h}} \delta_{S}\left[V_{N-1}\left(\tilde{\beta}_{h}\right)-V_{N-1}(0)\right]+\left(1-\frac{\beta_{N-1}^{R}}{\tilde{\beta}_{h}}\right) \delta_{B} \frac{\theta_{L}}{\Delta \theta}\left[U_{N-1}^{H}\left(\tilde{\beta}_{h}\right)-U_{N-1}^{H}(0)\right]>0 .
$$

Substituting $\beta_{N-1}^{R}$ we find that this inequality holds if and only if

$$
\frac{V_{N-1}\left(\tilde{\beta}_{h}\right)-V_{N-1}(0)}{V_{N-2}\left(\tilde{\beta}_{h}\right)-V_{N-2}(0)}>\frac{U_{N-1}^{H}(0)-U_{N-1}^{H}\left(\tilde{\beta}_{h}\right)}{U_{N-2}^{H}(0)-U_{N-2}^{H}\left(\tilde{\beta}_{h}\right)} .
$$

The LHS measures how strongly the seller's gain from learning increases with the number of periods. It is increasing in the seller's patience and therefore bounded from below by $\lim _{\delta_{S} \rightarrow \delta_{B}} \frac{V_{N-1}\left(\tilde{\beta}_{h}\right)-V_{N-1}(0)}{V_{N-2}\left(\tilde{\beta}_{h}\right)-V_{N-2}(0)}$. For $\delta_{S}=\delta_{B}$ continuation values must satisfy

$$
V_{K}(\beta)+U_{K}^{H}(\beta)=\beta \sigma_{K}+(1-\beta) \theta_{L} \sigma_{K}
$$

because total available surplus is divided between the seller and the (high type) buyer. Using this accounting identity for $K=N-1, N-2$ and $\beta=\tilde{\beta}_{h}$ we get

$$
\lim _{\delta_{S} \rightarrow \delta_{B}} \frac{V_{N-1}\left(\tilde{\beta}_{h}\right)-V_{N-1}(0)}{V_{N-2}\left(\tilde{\beta}_{h}\right)-V_{N-2}(0)}=\frac{\tilde{\beta}_{h} U_{N-1}^{H}(0)-U_{N-1}^{H}\left(\tilde{\beta}_{h}\right)}{\tilde{\beta}_{h} U_{N-2}^{H}(0)-U_{N-2}^{H}\left(\tilde{\beta}_{h}\right)} .
$$

Finally,

$$
\frac{\tilde{\beta}_{h} U_{N-1}^{H}(0)-U_{N-1}^{H}\left(\tilde{\beta}_{h}\right)}{\tilde{\beta}_{h} U_{N-2}^{H}(0)-U_{N-2}^{H}\left(\tilde{\beta}_{h}\right)} \geq \frac{U_{N-1}^{H}(0)-U_{N-1}^{H}\left(\tilde{\beta}_{h}\right)}{U_{N-2}^{H}(0)-U_{N-2}^{H}\left(\tilde{\beta}_{h}\right)}
$$

is equivalent to $\left(1-\tilde{\beta}_{h}\right)\left[U_{N-2}^{H}(0) U_{N-1}^{H}\left(\tilde{\beta}_{h}\right)-U_{N-1}^{H}(0) U_{N-2}^{H}\left(\tilde{\beta}_{h}\right)\right] \geq 0$ which holds because the buyer's gain in continuation value from the existence of one additional period is maximized when the seller is as soft as possible, i.e., when $\frac{U_{N-1}^{H}(0)}{U_{N-2}^{H}(0)} \geq \frac{U_{N-1}^{H}\left(\tilde{\beta}_{h}\right)}{U_{N-2}^{H}\left(\tilde{\beta}_{h}\right)}$. Hence, we can conclude that $f\left(\beta_{N-1}^{R}\right)>0$ and from properties (1)-(4) it follows that there must exist a unique $\beta_{N}^{R} \in\left(0, \beta_{N-1}^{R}\right)$ such that $V_{N}^{\text {sep }}>V_{N}^{\text {pool }}$ if and only if $\beta>\beta_{N}^{R}$. This completes the proof. 
Proof of Proposition 4. By induction over the number of periods $N$. Our analysis of the two-period model has shown that for $N=2$ the seller's optimal mechanism satisfies the properties described in Proposition 4. Assume that Proposition 4 holds for $N-1$. Suppose that there are $N$ periods of trade and the seller's prior is $\beta \in\left[0, \theta_{L}\right)$. Applying Lemma 1, the seller optimally sets $x_{h}^{*}=1$ and chooses $\left(x_{l}, \beta_{l}, \beta_{h}\right)$ to solve the reduced program in (15) with $K=N$. As this program is linear in $x_{l} \in[0,1]$ it must hold that $x_{l}^{*} \in\{0,1\}$, i.e., price-posting is optimal. For $x_{l}=1$ the seller's payoff becomes

$$
V_{N}^{\text {pool }}=\theta_{L} \sigma_{N}
$$

independently of the induced beliefs. For $x_{l}=0$ the constraint (16) requires that $\beta_{h}=1$ and it remains to solve the unconstrained program

$$
\max _{\beta_{l} \in[0, \beta]} \frac{\beta-\beta_{l}}{1-\beta_{l}}\left[\sigma_{N}-\delta_{B} U_{N-1}^{H}\left(\beta_{l}\right)\right]+\frac{1-\beta}{1-\beta_{l}} \delta_{S} V_{N-1}\left(\beta_{l}\right) .
$$

Consider first the case where $\beta \leq \beta_{N-1}^{S}$. Because $\beta_{l} \leq \beta$, our induction assumption implies that continuation values are given by $V_{N-1}\left(\beta_{l}\right)=\theta_{L} \sigma_{N-1}, U_{N-1}^{H}\left(\beta_{l}\right)=\Delta \theta \sigma_{N-1}$, and $U_{N-1}^{L}\left(\beta_{l}\right)=0$, i.e., continuation values are independent of $\beta_{l}$. As $\delta_{B} U_{N-1}^{H}\left(\beta_{l}\right)+$ $\delta_{S} V_{N-1}\left(\beta_{l}\right)<\sigma_{N}$ the objective in (47) is therefore decreasing in $\beta_{l} \in[0, \beta]$. It thus follows that for $\beta \leq \beta_{N-1}^{S}$ the solution to (47) is given by $\beta_{l}=0$ and it only remains to compare the payoff from pooling, $V_{N}^{\text {pool }}$, with the payoff from full separation given by

$$
V_{N}^{s e p}(\beta)=\beta\left[\sigma_{N}-\delta_{B} \Delta \theta \sigma_{N-1}\right]+(1-\beta) \delta_{S} \theta_{L} \sigma_{N-1} .
$$

Note that $V_{N}^{\text {sep }}(\beta)$ is strictly increasing in $\beta \in\left(0, \beta_{N-1}^{S}\right)$ with $V_{N}^{\text {sep }}(0)=\delta_{S} \theta_{L} \sigma_{N-1}<V_{N}^{\text {pool }}$ and

$$
\begin{aligned}
V_{N}^{\text {sep }}\left(\beta_{N-1}^{S}\right) & =V_{N-1}^{\text {pool }}+\beta_{N-1}^{S}\left[\delta_{B}^{N-1}-\delta_{B} \Delta \theta \delta_{B}^{N-2}\right]+\left(1-\beta_{N-1}^{S}\right) \delta_{S} \theta_{L} \delta_{B}^{N-2} \\
& =V_{N}^{\text {pool }}-\theta_{L} \delta_{B}^{N-1}+\beta_{N-1}^{S} \theta_{L} \delta_{B}^{N-1}+\left(1-\beta_{N-1}^{S}\right) \delta_{S} \theta_{L} \delta_{B}^{N-2}>V_{N}^{\text {pool }}
\end{aligned}
$$

where we have used the fact that $V_{N-1}^{\text {pool }}=V_{N-1}^{\text {sep }}=\beta_{N-1}^{S}\left[\sigma_{N-1}-\delta_{B} \Delta \theta \sigma_{N-2}\right]+(1-$ $\left.\beta_{N-1}^{S}\right) \delta_{S} \theta_{L} \sigma_{N-2}$ by the definition of $\beta_{N-1}^{S}$. Hence there exists a $\beta_{N}^{S} \in\left(0, \beta_{N-1}^{S}\right)$, given 
by the unique solution to $V_{N}^{\text {sep }}(\beta)=V_{N}^{\text {pool }}$, such that $V_{N}^{s e p}(\beta)>V_{N}^{\text {pool }}$ if and only if $\beta>\beta_{N}^{S}$. For $\beta \in\left(0, \beta_{N}^{S}\right]$ the seller pools by choosing $x_{l}^{*}=1, x_{h}^{*}=1$, and $\beta_{l}^{*}=\beta_{h}^{*}=\beta$ whereas for $\beta \in\left(\beta_{N}^{S}, \beta_{N-1}^{S}\right]$ the seller separates by choosing $x_{l}^{*}=0, x_{h}^{*}=1, \beta_{l}^{*}=0$, and $\beta_{h}^{*}=1$. Moreover, for $\beta>\beta_{N-1}^{S}$ separation might not be optimal but dominates pooling.

Proof of Corollary 2: From a comparison of the payoffs (46) and (48) in the $N$-period selling case we obtain

$$
\beta_{N}^{S}=\frac{\theta_{L}\left[1-\left(\delta_{S}-\delta_{B}\right) \sigma_{N-1}\right]}{1-\theta_{L}\left(\delta_{S}-\delta_{B}\right) \sigma_{N-1}}
$$

and in the limit $\beta_{\infty}^{S} \equiv \lim _{N \rightarrow \infty} \beta_{N}^{S}=\theta_{L} \frac{1-\delta_{S}}{1-\delta_{B}-\left(\delta_{S}-\delta_{B}\right) \theta_{L}}$. In analogy to $\sigma_{N}=\sum_{n=0}^{N-1} \delta_{B}^{n}$ define $\sigma_{N}^{S} \equiv \sum_{n=0}^{N-1} \delta_{S}^{n}$. For the $N$-period renting case we can determine an upper bound on $\beta_{N}^{R}$ by comparing the seller's payoff from pooling in (37) with his payoff from (full) separation given by (39) with $\beta_{h}^{*}$ set equal to 1 :

$$
\beta_{N}^{R} \leq \frac{\theta_{L} \delta_{B} \sigma_{N-1}}{\Delta \theta \delta_{S} \sigma_{N-1}^{S}+\theta_{L} \delta_{B} \sigma_{N-1}}
$$

Taking the limit we have that $\beta_{\infty}^{R} \equiv \lim _{N \rightarrow \infty} \beta_{N}^{R} \leq \theta_{L} \frac{\delta_{B}\left(1-\delta_{S}\right)}{\delta_{S}\left(1-\delta_{B}\right)-\theta_{L}\left(\delta_{S}-\delta_{B}\right)}$. Hence $\beta_{\infty}^{R}<\beta_{\infty}^{S}$ follows from

$$
\delta_{B}\left[\left(1-\delta_{B}\right)-\left(\delta_{S}-\delta_{B}\right) \theta_{L}\right]<\delta_{S}\left(1-\delta_{B}\right)-\theta_{L}\left(\delta_{S}-\delta_{B}\right) \Leftrightarrow \theta_{L}<1
$$

Moreover, $\beta_{N}^{R}<\beta_{N}^{S}$ if

$$
\begin{aligned}
\delta_{B} \sigma_{N-1}\left[1-\theta_{L}\left(\delta_{S}-\delta_{B}\right) \sigma_{N-1}\right] & <\left[1-\left(\delta_{S}-\delta_{B}\right) \sigma_{N-1}\right]\left[\Delta \theta \delta_{S} \sigma_{N-1}^{S}+\theta_{L} \delta_{B} \sigma_{N-1}\right] \\
\Leftrightarrow \delta_{B} \sigma_{N-1}\left(1-\delta_{S} \sigma_{N-1}^{S}\right) & <\delta_{S} \sigma_{N-1}^{S}\left(1-\delta_{S} \sigma_{N-1}\right)
\end{aligned}
$$

Note that each factor of the term on the RHS is larger than the corresponding factor on the LHS. Hence, if $1-\delta_{S} \sigma_{N-1}>0 \Leftrightarrow \delta_{S}<\frac{1}{\sigma_{N-1}}$ then $\beta_{N}^{R}<\beta_{N}^{S}$. This completes the proof. 


\section{References}

Bester, H., Strausz, R., 2001. Contracting with Imperfect Commitment and the Revelation Principle: The Single Agent Case. Econometrica 69, 1077-1098.

Bolton, P., Dewatripont, M., 2005. Dynamic Adverse Selection. In: Contract Theory. The MIT Press, Cambridge, MA.

Coase, R.H., 1972. Durability and Monopoly. J. Law Econ. 15, 143-149.

Deneckere, R.J., Preston McAfee, R., 1996. Damaged goods. J. Econ. Manag. Strategy $5,149-174$.

Freixas, X., Guesnerie, R., Tirole J., 1985. Planning under Incomplete Information and the Ratchet Effect. Rev. Econ. Stud. 52, 173-191.

Fudenberg, D., Tirole, J., 1983. Sequential Bargaining with Incomplete Information. Rev. Econ. Stud. 50, 221-247.

Gerardi, D., Maestri, L., 2016. The Ratchet Effect Implies No Learning: Dynamic Contracting without Commitment. Unpublished Manuscript.

Hart, O., Reny, P.J., 2015. Maximal Revenue with Multiple Goods: Nonmonotonicity and other Observations. Theor. Econ. 10, 893-922.

Hart, O., Tirole, J., 1988. Contract Renegotiation and Coasian Dynamics. Rev. Econ. Stud. 55, 509-540.

Krähmer, D., Strausz, R., 2015. Dynamic Mechanism Design. In: Börgers, T., An Introduction to the Theory of Mechanism Design. Oxford University Press, Oxford, England.

Laffont, J., Tirole, J., 1987. Comparative Statics of the Optimal Dynamic Incentive Contract. Eur. Econ. Rev. 31, 901-926.

Laffont, J., Tirole, J., 1988. The Dynamics of Incentive Contracts. Econometrica 56, $1153-1175$.

Laffont, J., Tirole, J., 1990. Adverse Selection and Renegotiation in Procurement. Rev. 
Econ. Stud. 57, 597-625.

Maestri, L., 2017. Dynamic contracting under adverse selection and renegotiation. J. Econ. Theory 171, 136-173.

Manelli, A., Vincent, D.R., 2007. Multidimensional mechanism design: Revenue maximization and the multiple-good monopoly. J. Econ. Theory 137, 153-185.

Mussa, M., Rosen, S., 1978. Monopoly and Product Quality. J. Econ. Theory 18, 301317.

Pavlov, G., 2011. Optimal Mechanism for Selling Two Goods. B.E. J. Theor. Econ. 11, 1935-1704.

Skreta, V., 2006. Sequentially Optimal Mechanisms. Rev. Econ. Stud. 50, 1085-1111.

Sobel, J., Takahashi, I., 1983. A Multistage Model of Bargaining. Rev. Econ. Stud. 50, 411-426.

Strulovici, B., 2017. Contract Negotiation and the Coase Conjecture: A Strategic Foundation for Renegotiation-proof Contracts. Econometrica 85, 585-616.

Thanassoulis, J., 2004. Haggling over Substitutes. J. Econ. Theory 117, 217-245.

Wang, G.H., 1998. Bargaining over a Menu of Wage Contracts. Rev. Econ. Stud. 65, 295-305. 